\title{
TEORÍA ENFERMERA EL CUIDADO DESDE UNA PERSPECTIVA PSICOSOCIAL
}

\author{
Begoña Carbelo Baquero*, Marta Romero llort**, Francisca Casas Martínez*, \\ Teresa Ruiz Ureña***, Sonsoles Rodríguez de la Parra*. \\ * Profesora Titular Interina en la Escuela de Enfermería y Fisioterapia de la Universidad de Alcalá. \\ ** Profesora Asociada de Ciencias de la Salud en la Escuela de Enfermería y Fisioterapia de la Universidad de Alcalá. \\ **:* Profesora Titular en la Escuela de Enfermería y Fisioterapia de la Universidad de Alcalá.
}

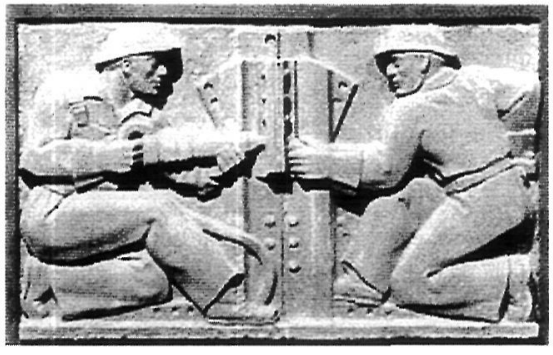

Correspondencia: Begoña Carbelo Baquero

c/ Jerez no 19

28230 Las Rozas.

Madrid

Este trabajo pretende hacer un análisis reflexivo de las aportaciones de distintas disciplinas en la proyección psicosocial de los cuidados enfermeros. La enfermería psicosocial es algo más que unos conocimientos psicológicos para profesionales de enfermería; las Ciencias Psicosociales, nos permiten abordar al ser humano desde el punto de vista holístico.

Las distintas escuelas o tendencias en enfermería, profundizan en el cuidado del ser humano y del entorno como componentes esenciales de la salud; desde estas líneas se analizan los aspectos psicosociales que permiten orientar las acciones enfermeras hacia el autocuidado de los pacientes y su familia, y describir, explicar y comprender el comportamiento humano no solo en la enfermedad sino en aspectos como calidad de vida, adaptación al entorno y el desarrollo de recursos para afrontar las distintas situaciones y alcanzar el bienestar.

Palabras clave: Cuidado, Enfermería, Ciencias Psicosociales, Holísmo, Autocuidado, Comportamiento, Bienestar.

\section{SUMMARY ${ }^{1}$}

This work intends to make a reflexive analysis of the different discipline contributions in the psychosocial projection of the nurse cares.

The psychosocial nursing is somewhat more than some psychological knowledge for professional of nursing; the Psychosocial Sciences permit us to approach to the human being from holistic point of view.

The different schools or trends in nursing, deepen in the care of the human being and of the environment as essential components of the health; from these lines are analyzed the different psychosocial aspects that permit to guide the nurse actions toward the self-care of patiens and their family, and to describe, to explain and to understand the human behavior not alone in the disease but in aspects as quality of life, adjustament to the environment and the resources development to face the different situations and to reach the welfare.

Keywords: Care, Nursing, Psychosocial Sciens, Nurse Schools, Holistic, Self-Care, Behavior, Welfare.

$\mathrm{El} \ll$ Summary» fue elaborado por los propios autores del artículo. 


\section{INTRODUCCIÓN}

Las Ciencias Psicosociales Aplicadas al Cuidado Enfermero, parten de unos conocimientos amplios, temáticamente heterogéneos, de fuentes muy diversas y a veces difíciles de delimitar. La psicosociología en Enfermería es de un pasado reciente. Los cuidados de la persona y la consideración de la misma como un ser global, en un entorno con el que interacciona constantemente, han sido los presupuestos básicos de la disciplina.

La influencia de las diversas maneras de entender la salud por parte de las Escuelas o Tendencias enfermeras, determinan las estrategias que han de utilizar los profesionales enfermeros y orientan el objeto de estudio de la disciplina. La proyección psicosocial en el cuidado enfermero nos permite conocer al ser humano desde la perspectiva de persona sana para poder comprender los aspectos del enfermar, fomentando actitudes que facilitan las relaciones y el crecimiento personal (Novel, 1991).

El elemento fundamental que conforma la Enfermería Psicosocial es el estudio de las manifestaciones del comportamiento suscitadas por la interacción de una persona con otras personas o por la mera expectativa de tal interacción (Jiménez Burillo, 1991).

En enfermería, se empieza a conceder importancia en el campo de la enfermería a materias relacionadas con las Ciencias Sociales y del Comportamiento en la confección de los planes de estudio de Enfermería, hace relativamente poco tiempo (Instituto Nacional de la Salud, 1991). Coincidiendo con la aparición de los Modelos teóricos de enfermería que hacen referencia especialmente a la perspectiva bio-psico-social y el cambio en la concepción de la salud en la misma línea, empieza a tomarse en cuenta que las teorías y modelos contienen elementos que han de estudiarse específicamente para un mejor entendimiento de los mecanismos que originan las desviaciones de la salud y dar un enfoque adecuado y te-

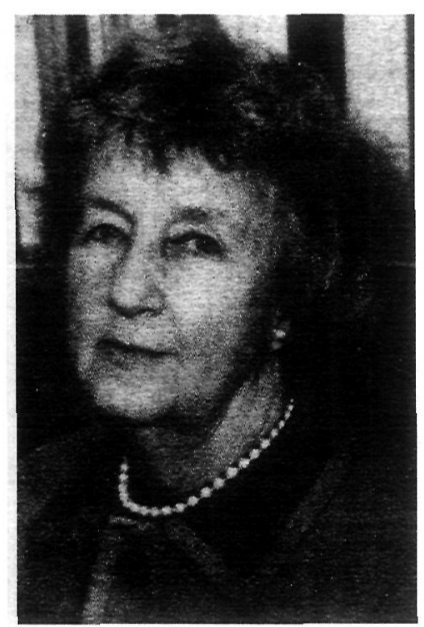

Virginia Henderson rapéutico a los cuidados de enfermería siguiendo unas directrices para la planificación de las enseñanzas de Enfermería que tengan en cuenta en el desarrollo del currículum básico las siguientes cuestiones: Procesos psicológicos básicos, psicología del desarrollo, psicología de la salud (Latorre Postigo, 1995).

Según la expresión de la conceptualización de la enfermería para D. Orem
(Orem, 1993). La enfermería es un servicio de salud especializado y se distingue de otros servicios humanos por su foco de atención en las personas con incapacidades para la continua provisión de la cantidad o calidad de cuidados en un momento específico que son reguladores de su propio funcionamiento y desarrollo (...) concepto generalizador que se apoya en conceptos relacionados con los aspectos psicosociales de la enfermería en general. En la Escuela de Enfermería de la Universidad de Alcalá, existe la conciencia de que los futuros profesionales de la salud, ahora estudiantes de Enfermería, necesitan una visión general, actual y pormenorizada en muchos casos de los aspectos relacionados con las Ciencias Psicosociales para conocer al ser humano con el que han de establecer una relación de ayuda desde todos los aspectos que abarca la persona potenciando el autocuidado y reforzando las propias capacidades.

\section{OBJETO DE ESTUDIO DE LAS CIENCIAS PSICOSOCIALES APLICADAS AL CUIDADO ENFERMERO}

Entre los temas de interés que se estudian desde el enfoque psicosocial, están fenómenos como la percepción, aprendizaje, personalidad, actitudes, motivación, sentimientos y emociones, prejuicios, influencia social, la familia, la relación de ayuda en el cuidado; aspectos que se estudian en el currículo básico y que son de marcada importancia para centrar los cuidados de las personas y comprenderlos, estén sanos o enfermos en su relación con las demás personas (E.U. de Enfermería y Fisioterapia, 1996). La enfermería psicosocial, se apoya fundamentalmente en las tres diciplinas que le dan nombre: Enfermería, Psicología y Sociología. Unas veces el cuidado de los individuos centra el interés en variables psicológicas como sentimientos, emociones, actitudes, personalidad..., otras veces se apoya en variables socioculturales como cultura, sociedad, grupos, familia, influencia, liderazgo, pero siempre desde una visión global de la persona en interacción con el entorno y para dispensar unos cuidados en todas las situaciones en un mundo en constante evolución. La enfermería requiere la aplicación del entendimiento, conocimientos y habilidades específicas de la disciplina enfermera, así como integrar conocimientos de otras ciencias como las Ciencias Humanas y las Ciencias Sociales (Durán, 1994), y es en este apartado donde entra de lleno las aplicaciones de la Enfermería Psicosocial.

\section{APORTACIONES DE OTRAS CIENCIAS A LAS CIENCIAS PSICOSOCIALES APLICADAS AL CUIDADO ENFERMERO}

Son muchas las aportaciones de otras teorías a las C. Psicosociales Aplicadas a Enfermería, pero son de marcada importancia las teorías más amplias, como las dinámicas, o humanísticas, que parten de las experiencias del ser humano para explicar los comportamientos y profun- 
dizan en aspectos más individuales que generales, acentuando la importancia de la subjetividad, experiencias personales, creatividad, desarrollo personal (VVAA, 1995). La intervención de la enfermera desde la perspectiva psicosocial, es la actividad centrada en ayudar a la persona, familia o grupo, a hacer frente a los cambios y a las crisis de una manera adaptativa y a potenciar los recursos de la persona y utilizar las experiencias vitales como elementos de crecimiento personal (E.U. de Enfermería y Fisioterapia, 1996).

Las Teorías del Modelo Biologicista:consideran los problemas emocionales y del comportamiento como patologías orgánicas al igual que otras enfermedades. Entre las distintas líneas de estudio, parece de interés destacar la genética, la neuroquímica, la neurofisiología y los neurotransmisores, estos dos últimos son importantes desde nuestro punto de vista, ya que está demostrado que muchas situaciones relacionadas con el estrés estimulan sustancias que influyen en el estado de ánimo.

Actualmente está en estudio el potencial de unas sustancias producidas por el cerebro llamadas endorfinas y que son el vehículo de la euforia y alivio del dolor y actúan como mensajeros bioquímicos. Las endorfinas son poderosos analgésicos bioquímicos segregados por el cerebro y constituyen uno de los descubrimientos científicos más importantes de éste siglo. El masaje, los deportes, la música, la relajación, hacer lo que apetece... hace que el cerebro fabrique endorfinas y hace sentir bien a las personas (Lawson, 1995; Goldberg, 1989), y los elementos nombrados están al alcance y posibilidades de la enfermería en base a los conocimientos aportados por la enfermería psicosocial.

Las Teorías conductistas: toman en cuenta la personalidad como una acumulación de patrones de conducta adquiridos, gobernados por los principios de aprendizaje y sujetos a influencias ambientales.

Esta teoría de la que Skiner es uno de los más insignes representantes, para los cuidados puede ser interesante en el sentido de permitir explicar algunas conductas adquiridas en los niños y problemas específicos.

La Teoría Cognitiva: A partir de los años 50 surge una corriente, la cognitiva que es contrapuesta al conductismo. Empieza a vislumbrarse que algo está cambiando en la época y se centra el interés en procesos cognitivos superiores como la percepción, atención, pensamiento, lenguaje, memoria... Hay una clara influencia de Piaget y la Escuela de Ginebra.

Jean Piaget: Fue el gran impulsor de esta corriente con sus estudios sobre la percepción y la inteligencia. Considera que la personalidad humana evoluciona a partir de la interacción de las funciones afectivas e intelectuales. La maduración se da con los procesos de asimilación y acomodación.

Teorías Dinámicas: Se derivan de las ideas de las ideas de Sigmund Freud (1915), Toda conducta humana está determinada, ya que se origina a partir de las experiencias infantiles de cada persona, y es inconsciente, puesto que la verdadera motivación no suele ser conocida por el individuo. El motor que determina la conducta del ser humano es la energía psíquica denominada por Freud como «libido», Esta energía depende de los impulsos fisiológicos e instintivos, hambre, sed, deseo sexual, es la liberación de la tensión mediante la experimentación del placer.

La personalidad humana puede ser comprendida, desde tres estructuras teóricas: ello, yo y superyó. La ansiedad es el resultado de la lucha continua entre el ello y el superyó, que deben ser mantenidos bajo control por él yo, cuya integridad está continuamente amenazada. El yo intenta evitar su destrucción mediante mecanismos de defensa. El inconsciente es el lugar de los impulsos innatos, los deseos, sentimientos y deseos reprimidos.

De gran influencia en autoras como H. Peplau, las teroriás dinámicas aportan a la Enfermería Psicosocial, puntos de vista flexibles del ser humano, tomando en cuentas fenómenos no observables para explicar la conducta.

Teorias humanistas: El enfoque humanista surge entre las décadas de los años 40 y 60, como reacción a las teorías conductistas que no consideran los procesos internos y la experiencia individual, y a las psicoanalíticas por tener criterios manifiestamente pesimistas y mecanicistas.

La psicología humanista considera a la persona la fuente más fiable de conocimiento respecto a sus propias cápacidades, recursos y características. El modelo humanista tiene una confianza optimista en la naturaleza positiva del hombre.

Su principal objetivo es el crecimiento de la persona. Basa sus valores en la libertad personal, responsabilidad del individuo, la igualdad de oportunidades y la protección del entorno.

Sus principales representantes son :

Abraham Maslow: De gran influencia sobre la enfermería. Maslow veía a la persona como portadora de una naturaleza innata esencialmente buena. Consideraba que la persona tiende a su autorealización. La persona tiene que satisfacer unas necesidades fisiológicas y de seguridad para pasar a otras más elevadas como el amor, la autoestima para llegar al final de la pirámide donde se sitúa la necesidad de autorrealización. La ausencia de satisfacción de necesidades en los niveles más bajos bloquea la posibilidad de crecimiento personal.

Carl Rogers: (1961) Es el fundador de la llamada Terapia centrada en el cliente, pretende que la persona recupere sus capacidades de autorrealización.

La herramienta para la ayuda es la relación. Considera que el terapeuta debe tener tres actitudes bási- 
cas en la relación de ayuda que son: empatía, apoyo incondicional y congruencia.

Estas teorías han tenido gran influencia en la Escuela del Caring.

Teoría psicosocial del desarrollo: La Escuela de las Necesidades parte de éste autor además de Maslow par elaborar sus teorías : Erik Erikson, psicoanalista norteamericano, hizo una de las contribuciónes más importantes en el área del desarrollo de la personalidad centrándose en el Yo.

La estructuración de un Yo saludable es un proceso gradual que se conforma etapa tras etapa del ciclo vital, basado en la experiencia de salud social y solidaridad cultural, el cuidado se centra en cada etapa del desarrollo con sus características que la definen.

Teoría General de Sistemas: La teoría general de los sistemas, formulada por Von Bertalanffy, propone la integración de los sistemas personal, interpersonal y social que interactúan con otros sistemas, y estas interrelaciones tienen sus efectos dentro del individuo y de la unidad familiar. Estas teorías influyen en las Escuela de los Efectos deseables.

Teorías de la Comunicación: Desde cualquier perspectiva que se aborde, resulta de indudable importancia, ya que, la comunicación es el medio a través del cual la persona establece relaciones; para los enfermeros, esta importancia cobra mayor interés como elemento básico de la relación terapéutica. Para Cherry (King, 1984) la comunicación «es un proceso dinámico, fundamental para la existencia, el crecimiento, el cambio y la conducta de todos los seres vivos, ya sean individuos u organizaciones». La Escuela de Palo Alto (Waztlawick, 1985), en los años 50 promovió un avance importante en el campo de la comunicación humana, iniciaron su trabajo con el estudio de la comunicación entre los pacientes y sus familias y elaboraron una Teoría de la Comunicación Humana, considerada en el campo de la enfermería como

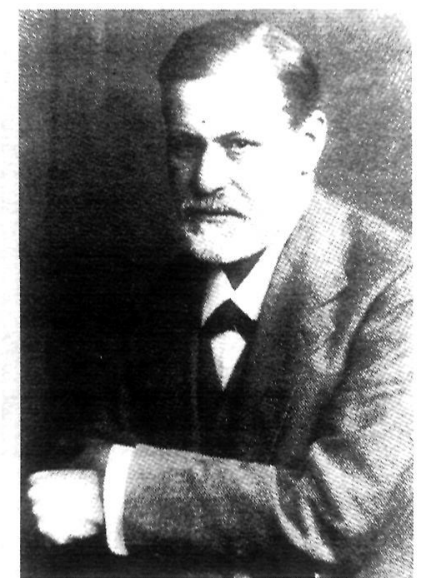

Carl Rogers de influencia en todas las escuelas.

Teorías sociológicas: Se basan generalmente en que toda conducta humana, por atípica que parezca es normal, siendo la sociedad la que califica algunos individuos como enfermos mentales, por el simple hecho de desviarse de los que considera socialmente aceptable.

\section{David Cooper} (1971) El fundamento teórico procede de C. Marx. El hombre como ser social no puede vivir fuera de una sociedad. Sin embargo al construir la sociedad se producen fenómenos sociales tales como el poder, el control, que escapan a la idea genuina de sociedad y actúan sobre el sujeto de forma alienante impidiéndole que desarrolle su propia conciencia pensante individual.

\section{EL CUIDADO DESDE EL PUNTO DE VISTA PSICOSOCIAL EN LAS DIFERENTES ESCUELAS ENFERMERAS}

Después de la década de los setenta, se observa una gran preocupación de las enfermeras por los aspectos humanísticos y filosóficos del cuidado. Cuidar a una persona supone el reconocimiento de sus valores culturales, sus creencias y convicciones (Leininger, 1991: 21-23). Desde ésta perspectiva Rogers invita a las enfermeras a hacer investigaciones hacia los cuidados de salud creativos que pueden ser aplicados en la tierra y más allá de nuestro planeta (Rogers, 1989: 112-116); Gortner señala que la disciplina enfermera es concebida hoy como una ciencia del comportamiento y de la salud de la persona en todas las edades de la vida, es decir una ciencia que incluye, factores biológicos, comportamentales y sociales así como una definición de los resultados esperados y de los indicadores de salud. Para dispensar cuidados enfermeros es cada vez más necesario comprender los procesos, los problemas y las situaciones de las personas de todas las edades, en todas las situaciones de la vida y en un mundo en constante evolución (Gortner, 1983: 1-8; Polit \& Hungler, 1991).

La disciplina enfermera ha evolucionado y con ella las necesidades de formación de las enfermeras, en el contexto de acontecimientos históricos y movimientos sociales. Por ello es necesario que dada la multiplicidad de las situaciones de salud, se orienten los cuidados reconociendo al ser humano en el seno de la sociedad y en todas sus dimensiones (Kerouac, 1996). Según las bases filosóficas y científicas las distintas concepciones de la disciplina enfermera se pueden agrupar en seis escuelas : Escuela de las necesidades, de la interacción, de los efectos deseados, de la promoción de la salud, del ser humano unitario y del caring (Kerouac, 1996). Partimos inicialmente de que todas están orientadas hacia la persona, con lo que unos conocimientos amplios

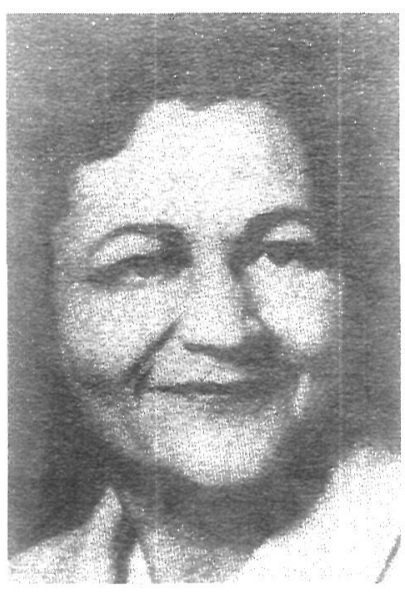
Imogene King 
Escuela se amplía a la familia que aprende de sus propias experiencias de salud. Según Moyra Allen, el primer objetivo de los cuidados enfermeros es la promoción de la salud, mediante el mantenimiento, el fomento y el desarrollo de la salud de la familia y de sus miembros por la activación de sus procesos de aprendizaje. La enfermera desempeña un rol facilitador, estimulador del aprendizaje en el cuidado de los pacientes.

5. Escuela del Ser Humano Unitario: Se sitúa en el contexto del ser humano hacia el mundo y el paradigma de la transformación. En el cuidado, la enfermera y la persona actúan juntas en consenso. Esta Escuela se basa en concepciones humanistas y fenomenológicas del ser humano, en donde la enfermera, suscita la participación.

6. Escuela del Caring: Watson en 1985 (Watson 1985) propone un caring que sea a la vez humanista y científico, considerando aspectos del ser humano como la espiritualidad, y la cultura. Los trabajos de Carl Rogers, inspiran e influencian la visión de esta escuela en la que la relación transpersonal se impregna de coherencia, empatía y calor humano. El objetivo de los cuidados enfermeros es el de ayudar a la persona a conseguir el más alto nivel de armonía entre su alma su cuerpo y su espiritu. La enfermera percibe y siente lo vivido por la otra persona y responde a ello de forma que le permite exteriorizar sus sentimientos.

\section{CONCLUSIONES}

El desarrollo de la Enfermería Psicosocial, va parejo al desarrollo de las escuelas enfermeras. Los primeros modelos incorporan postulados que tienen que ver con los aspectos psicosociales del ser humano. Es necesario ir incorporando conocimientos profundos que permitan cuidar a las personas con una metodología científica. Son necesarias las aportaciones de las distintas disciplinas, en éste caso de más relevancia, la Psicología y las Sociología al conocimiento enfermero. La Psicología dota a la enfermería de conocimientos que le permiten conocer al hombre ya sea sano o enfermo situado en su entorno influyente. La Sociología permite a la enfermera incorporar unos conocimientos que ayudan a comprender a los individuos en relación a otros individuos, grupos, familia, cultura y sociedad y poder intervenir. Pero lo que en definitiva aporta la Enfermería Psicosocial a los futuros profesionales es que sean además de profesionales ilustrados, hábiles en la utilización de la razón, en todos los ámbitos de la vida, para ayudar a las personas a alcanzar la autonomía en su autocuidado.

\section{BIBLIOGRAFÍA}

Durán, M. (1994) Modelo ideológico y de organización de la estructura docente en la Escuela de Enfermería. Universidad de Alcalá de Henares. Madrid.

Goldberg, J. (1989) Las Endorfinas. Editorial Gedisa. Barcelona.
Gortner, S.R. (1983) The History anfd Philosophy of Nursing Science. Advance of Nursing Science 5 /2, $1-8$.

Jiménez Burillo, F. (1991) Psicología Social. Ediciones Academicas. Uned. Madrid.

Instituto Nacional de la Salud (1991) Metodología de la Enseñanza de Enfermería. E. U. La Paz., Madrid.

King I. M. (1984) Enfermería como Profesión. Limusa. Mexico. Kerouac, S. et al (1996). El Pensamiento Enfermero. Masson, S.A. Barcelona.

Latorre Postigo, J.M. (1995) Ciencias Psicosociales Aplicadas I. Editorial Sintesis. Madrid.

Lawson, J. (1995) Endorfinas. La droga de la felicidad. Ediciones Obelisco. Barcelona.

Leininger, M.M. (1991) Transcultural Care Principles, Human Rights and Ethical Considerations. Journal of Transcultural Nursing 3/1, 21-23.

Meleis, A.I. (1991) Theoretical Nursing: Development and Progress, $2^{\text {a }}$ edic. Philadelphia, Lippincott.

Marriner-Tomey, A. (1991) Nursing Theorists and their work. $2^{a}$ edic. St. Louis. The C.V. Mosby Company.

Novel G. et al (1991) Enfermería Psicosocial II. Serie Manuales de Enfermería. Salvat editores, S.A. Barcelona.

Orem, D. (1993) Modelo de Orem. Conceptos de Enfermería en la práctica. Masson Salvat. Barcelona.

(E.U. Enfermería y Fisioterapia) (1996) Programa de Ciencias Psicosociales. Universidad de Alcalá. Alcalá de Henares.

Polit, D.F. \& Hungler, B.P. (1991) Nursing Research : Principles and methods. $4^{\mathrm{a}}$ edic, PA. Lippincott.. Philadelphia

Rogers, M.E. (1989) Creating a Climate for the implementation of a Nursing Conceptual Framework. The Journal of Continuing Education in Nursing 20/3, 112-116.

Roy, C. (1986) Introduction aux soins infirmiers: un modèle de l'adaptation, traducción de Introduction to Nursing: An adaptation model 1984, par Louise Betrger, Gaëtan Morin editeur, Montreal.

Roy, C. \& Andrews, H.A. (1991) The Roy Adaptation Model: The Definitive Statement, Appleton \& Lange, Norwalk.

(VVAA) (1995) Master de Enfermería. Masson, S.A. Barcelona.

Watson, J. Nursing (1985) The Philosophy and Science of Caring, $2^{\mathrm{a}}$ edic., Associated University Press, Boulder, Colorado.

Waztlawick, P. et al (1985) Cambio. Herder, Barcelona. 